\title{
Universiteit
}

Leiden

The Netherlands

\section{Quantum smectic as a dislocation Higgs phase}

Cvetkovic, V.; Zaanen, J.

\section{Citation}

Cvetkovic, V., \& Zaanen, J. (2006). Quantum smectic as a dislocation Higgs phase. Physical Review Letters, 97(4), 045701. doi:10.1103/PhysRevLett.97.045701

Version: $\quad$ Not Applicable (or Unknown)

License: $\quad$ Leiden University Non-exclusive license

Downloaded from: https://hdl.handle.net/1887/71389

Note: To cite this publication please use the final published version (if applicable). 


\title{
Quantum Smectic as a Dislocation Higgs Phase
}

\author{
V. Cvetkovic* and J. Zaanen ${ }^{\dagger}$ \\ Instituut Lorentz voor de theoretische natuurkunde, Universiteit Leiden, P.O. Box 9506, NL-2300 RA Leiden, The Netherlands
}

(Received 27 April 2006; published 24 July 2006)

\begin{abstract}
The theory describing quantum smectics in $2+1$ dimensions, based on topological quantum melting is presented. This is governed by a dislocation condensate characterized by an ordering of Burger's vector and this "dual shear superconductor" manifests itself in the form of a novel spectrum of phononlike modes.
\end{abstract}

DOI: 10.1103/PhysRevLett.97.045701

Different from classical liquid crystals [1,2], the quantum smectic and nematic type orders occurring at zero temperature are far from understood. These came into focus recently, motivated by empirical developments in high $T_{c}$ superconductivity and quantum-Hall systems [3]. Fundamentally, it is about the partial breaking of the symmetries of space itself, and on the quantum level this might carry consequences which cannot be envisaged classically.

The "most ordered" liquid crystal is the smectic, which can be pictured as lines in two dimensions (or layers in three dimensions) of liquid forming a periodic array in one spatial direction. Emery et al. [4] (for doped Mott insulators) and MacDonald and Fisher [5] (for quantumHall systems), delivered proof of principle that things are different on the quantum level by showing that a twodimensional quantum system can organize spontaneously into an array of one-dimensional metals. Here we will present a description of the quantum smectic which is complementary to these earlier works. It rests on KramersWannier duality [6,7], the field-theoretical fact that the disordered state (the smectic) corresponds to an ordered state (in fact, the Higgs phase) formed from the topological excitations (dislocations) of the ordered state (the crystal), and as such it can be viewed as a quantum extension of the famous Nelson-Halperin-Young [8] theory of twodimensional melting.

The theory is completely tractable for a system of bosons living in the $2+1$ D Galilean invariant continuum [9], in the limit that all characteristic length scales are large compared to the lattice constant. The outcome is a spectrum of propagating long-wavelength collective modes [10], which should have a universal status in the scaling limit. Before discussing the theory, let us first present this mode spectrum. The quantum smectic in $(2+1) \mathrm{D}$ is characterized by a "crystalline" and an orthogonal "liquid" direction [see Fig. 1(d)]. For simplicity we assume that the quantum smectic is associated with a reference crystal described by isotropic quantum elasticity (e.g., a hexagonal crystal) characterized by just a shear $(\mu)$ and compression $(\kappa)$ modulus, and a mass density $\rho$, such that the longitudinal and transversal phonon velocities are given
PACS numbers: 64.60.-i, 71.10.Hf, 71.27.+a, 74.20.Mn

by $c_{L}=\sqrt{(\kappa+\mu) / \rho}$ and $c_{T}=\sqrt{\mu / \rho}$. For modes propagating exactly along either the fluid $[\eta=0 \bmod \pi$, i.e., Figs. 1(a) -1 (c)] or solid [ $\eta=\pi / 2 \bmod \pi$, i.e., Figs. 1(e)$1(\mathrm{~g})]$ directions transversal and longitudinal motions decouple. Let us first focus on the solid direction. The longitudinal mode [Fig. 1(b)] vibrates the "leftover" lattice and behaves like a phonon; it actually propagates with $c_{L}$, showing that this mode is set by the shear rigidity of the reference crystal. The transversal mode [Fig. 1(c)], corresponding to "sliding motions" of the "liquid lines" relative to each other, is very different from a phonon: there is no massless mode and we find instead a single massive "dual Higgs photon" [Fig. 1(a)] which is the fingerprint of the "true" liquid as we will explain later. This implies the absence of a reactive elastic response, and our quantum smectic can be understood as "an array of independent one-dimensional quantum liquids", in the same sense as the Kivelson-Lubensky sliding phases [4].

Let us now turn to the modes propagating along the fluid direction [Fig. 1(g)]. The longitudinal mode [Fig. 1(e)] looks like a compressional wave in the liquid, but it is again indistinguishable from the longitudinal phonon of the reference crystal. The transversal wave in the liquid direction [Fig. 1(f)] is clearly not fluctuating the lattice
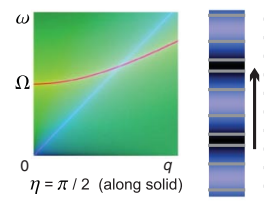

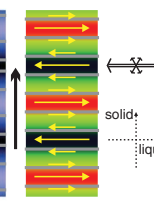

b) c) d)

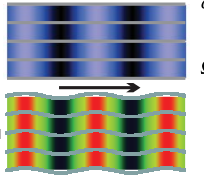

e, f)

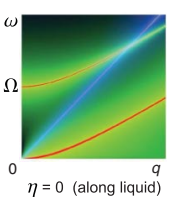

FIG. 1 (color). The elastic response (strain spectral functions) of a quantum smectic as function of momentum $(q)$ and frequency $(\omega)$ in the two special solid (a) and liquid (g) direction, as determined by the orientation of the condensate Burgers vectors (d). The longitudinal phonons (blue) are unaffected, corresponding either to (b) compression of layers along the solid direction or to (e) density waves in the liquid direction. The transversal response (red) is gapped along the solid direction due to the decoupling of the layer motion (c). Along the liquid direction (f), shear rigidity is screened, but the transversal motions of the layers results in a massless quadratic mode. 
directly, but it is neither a pure motion of the liquid. The outcome is remarkable: besides the dual Higgs photon, the fingerprint of the liquid, we find a mode with a quadratic dispersion: $\omega=\lambda_{S} c_{T} q^{2}$, where $\lambda_{S}$ is the shear penetration depth, the characteristic length of crystal correlations in the liquid, associated with the "shear Higgs mass" by $\Omega=$ $c_{T} / \lambda_{S}$. These quadratic modes are of course well known from the classical smectics $[1,2,11]$, but they are also consistent with the findings in the quantum-Hall smectic [5]. As will become clear, they arise in the present context in a quite surprising way.

What happens when one deviates from either the liquid or solid direction? The transversal and longitudinal modes now couple and at arbitrary angles a "longitudinal-like" mode is present with a velocity decreasing from $c_{L}$ to, amusingly, a purely compressional velocity $c_{\kappa}=\sqrt{\kappa / \rho}$ at $\pi / 4$ [Fig. 2(b)]. In the transversal-like response one finds always the dual Higgs photon. However, deviating infinitesimally from the solid direction $(\eta=\pi / 2)$ one finds immediately a new massless pole in the spectrum with a velocity $c_{1} \approx c_{T} \eta \sqrt{2(1+\nu)}$, demonstrating that shear rigidity becomes finite [Fig. 2(a)]. This mode persists at all intermediate angles, with a velocity going through a maximum at $\eta=\pi / 4$. Upon approaching the liquid direction ( $\eta=0$, Fig. 2(c)] it continues to be the only "transversallike" massless mode which turns into the quadratic mode [Fig. $1(\mathrm{~g})]$ precisely at $\eta=0$.

To the best of our knowledge, the massles excitations amount to a novel enumeration of the Goldstone sector of the quantum-smectic state. The way we derived it might be surprising: it can be viewed as the observable "shadow" of an underlying order which we call the dual shear superconductor. It is about a duality construction which is a close sibling of the well-known vortex duality in $(2+1) \mathrm{D}$ [12-14]: the quantum disordered superfluid (Mottinsulator) corresponds to a Bose condensate of vortices. Since the long range interactions between the vortices are indistinguishable from electromagnetic interactions, this dual condensate is a (gauged) superconductor. It is even a

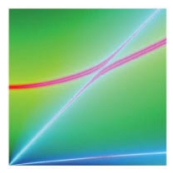

a)

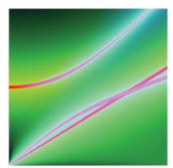

b)

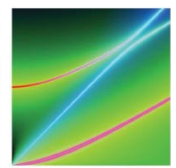

c)
FIG. 2 (color). The long-wavelength modes at intermediate values of the propagation angle $\eta$. (a) Close to the "solid" direction $(\eta=14 \pi / 30)$ the longitudinal phonon mode and massive shear photon of Fig. 1(a) are coupled, but in addition a massless mode has appeared with a velocity and pole strength in the strain propagator increasing $\propto \eta$. (b) Halfway $(\eta=\pi / 4)$, one finds two massless modes with velocities $c_{T}$ and the compressional velocity $c_{\kappa}$, as well as the massive shear photon. (c) Close to the "liquid" direction $(\eta=\pi / 30)$, the quadratic mode of Fig. 1(g) acquires a linear dispersion at small momenta. relativistic Higgs condensate: the particle and hole excitations of the Mott insulator are identified with the "massive Goldstone" and "longitudinal" photon of the relativistic theory [14]. In dual elasticity the role of electromagnetic fields is taken by the stress fields, hence the dual condensate of dislocations is a (gauged) superconductor where (dual) shear stress becomes short ranged.

Turning to the quantum crystal, the breaking of translations implies shear rigidity and the translational topological charge is the Burgers vector of the dislocation [15]. Disclinations take care of rotations and in topological language a (quantum) liquid crystal is a medium where dislocations have proliferated while disclinations stay massive, as pointed out a long time ago in the classical context by Nelson, Halperin, and Young [8]. This classic work deals with nematic ("hexatic") order and how does one fit in smectics? In this regard we profit from a very recent theoretical advance. In the construction of the dislocation condensates one has room to play with the vectorial topological charges, and according to Bais and Mathy [16], the usual recipe for spontaneous symmetry breaking based on Lie groups does not suffice to classify defect condensates. Instead one needs Hopf symmetry, a larger mathematical structure keeping track of symmetry and topology simultaneously, with the "quantum-double" Hopf symmetry being the one of relevance to liquid crystalline orders [16]. Their bottom line is illustrated in Fig. 3: consider for simplicity the hexagonal crystal breaking the symmetry of $2 \mathrm{D}$ Euclidean space $\mathbb{R}^{2} \ltimes O(2)$ to $\mathbb{Z}^{2} \ltimes D_{6}$. The Burgers vectors can take values along any of the six directions in the hexagonal lattice. According to the quantum-double machinery, one possibility is that the Burgers vectors are populated with equal probability along all six directions

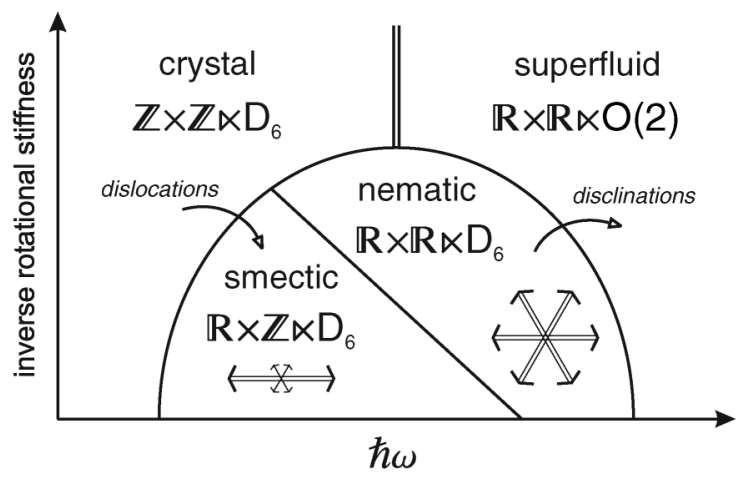

FIG. 3. The topology of the zero temperature phase diagram as suggested by "quantum-double Hopf symmetry breaking" [16]. The amount of quantum disorder (i.e., coupling constant $\hbar \omega$ ) is represented on the horizontal axis; the vertical axis carries the inverse rotational stiffness, a parameter whose smallness prohibits the proliferation of disclinations $[10,17]$ that would lead to the isotropic superfluid phase. The nematic or "quantum hexatic" state with the occupation of all allowed Burgers vectors with equal probability while the smectic corresponds in this topological scheme to a preferential occupation one particular dislocation-antidislocation pair. 
and the overall symmetry of this state is $\mathbb{R}^{2} \ltimes D_{6}$, the hexatic nematic state, where all translations are restored but rotations are broken. However, it is also allowable to condense dislocation-antidislocation pairs in one particular direction and the symmetry of this state is $\mathbb{R} \times \mathbb{Z} \ltimes D_{6}$, which is the symmetry of the smectic. Hence, smectic order is part of the repertoire of topological melting, and at zero temperature it should be located in between the crystal and the hexatic (Fig. 3).

The above amounts to a symmetry classification and next is the construction of the theory describing the dynamics in $(2+1) D$. Since dislocations are Abelian defects, this follows the pattern of vortex duality resulting in a dual gauge theory. Adapting the methods developed by Kleinert in the 1980s for the 3D classical case [17] we derived the $(2+1) \mathrm{D}$ theory in an earlier paper [9], but this contains some technical errors, which are serious enough to obscure the physics: the neglect of the condensate dynamics and a flawed gauge fix. These are now repaired [10], and we will present here a summary of this earlier work, to discuss in detail the correct treatment of the dual shear superconductor.

Consider a hexagonal crystal in $(2+1) \mathrm{D}$ with a longwavelength action coinciding with isotropic quantum elasticity: the usual theory in terms of the strains $\partial_{a} u^{b}\left(u^{b}\right.$ 's are the atomic displacements) and an added kinetic energy $(\rho / 2)\left(\partial_{\tau} u^{a}\right)^{2}$. In terms of the stress field $\sigma_{\mu}^{a}(\mu=x, y$, $\tau$, and $a=x, y$ ), dual to the strains $\partial_{\mu} u^{a}$, the Lagrangian reads in $(2+1) \mathrm{D}$ Euclidean space-time $[9,17]$,

$$
\mathcal{L}_{\text {gauge }}=\frac{1}{2} \sigma_{\mu}^{a} C_{\mu \nu a b}^{-1} \sigma_{\nu}^{b},
$$

where $\sigma_{\tau}^{a}$ corresponds to the momenta dual to the velocities $\partial_{\tau} u^{a}$, with inverse coupling constant $C_{\tau \tau a b}=\delta^{a b} / \rho$. The conservation of stress implies $\partial_{\mu} \sigma_{\mu}^{a}=0$ and this can be imposed in $(2+1) \mathrm{D}$ by expressing the stress fields in terms of stress gauge fields [17] $B_{\mu}^{a}$,

$$
\sigma_{\mu}^{a}=\epsilon_{\mu \nu \lambda} \partial_{\nu} B_{\lambda}^{a} .
$$

Hence, the theory describing acoustic phonons can be formulated as a Maxwell theory containing two "flavored" $(a=x, y) U(1)$ gauge fields - these photons describe the capacity of the solid to carry elastic forces. Imposing the Ehrenfest constraint $\left(\sigma_{x}^{y}=\sigma_{y}^{x}\right)$, one ends up with a Coulomb force between static sources and two propagating photons, coinciding with the longitudinal and transversal phonon of the $(2+1) \mathrm{D}$ medium $[9,10]$. The sources of these stress gauge fields are the nonintegrabilities associated with the translational part of the displacement fields $[9,17]$,

$$
\mathcal{L}_{\text {int }}=i B_{\mu}^{a} J_{\mu}^{a}, \quad J_{\mu}^{a}=\epsilon_{\mu \nu \lambda} \partial_{\nu} \partial_{\lambda} u^{a} .
$$

These dislocation currents can be factorized as $J_{\mu}^{a}=b^{a}$ $\mathcal{J}_{\mu}$, the world lines of dislocations with Burgers vector $\vec{b}=\left(b_{x}, b_{y}\right)$, implying $B_{\mu}^{a} J_{\mu}^{a} \rightarrow\left(b^{a} B_{\mu}^{a}\right) \mathcal{J}_{\mu}$. This structure is quite similar to vortex duality: the dislocations are "charged" particles (like the vortices) minimally coupled to gauge fields mediating the long-range interactions, except that these fields are now stress gauge fields, while the "Burgers" charge is vectorial.

The kinematics of dislocations is unusual because of the "glide constraint", the fact that dislocations only propagate in the direction of their Burgers vectors [15], implying that the spacelike components of the dislocation currents form symmetric tensors $J_{y}^{x}-J_{x}^{y}=0[9,18]$. A ramification is that dislocations only carry shear, and no compressional gauge charge. The effect is that only shear rigidity is destroyed by the dislocation condensate, while it still carries sound $[9,18]$. Glide plays an especially interesting role in the quantum smectic: although the dislocations form a $(2+1) \mathrm{D}$ condensate, the Burgers vectors are oriented in the liquid direction and only in this direction "diamagnetic" currents occur, screening the shear stress. This fact is largely responsible for the dichotomy highlighted in Fig. 1.

We overlooked in our previous work [9] the fact that the velocity scale of the dislocation condensate coincides with the transversal phonon velocity, playing the role of light velocity: the dual shear superconductor is a relativistic "Higgs phase". This is implied by the effective Lorentz invariance of the starting theory [14], where space and time derivatives appear on equal footing such that the phonon velocity also governs the dynamics of the defects [15]. Having collected all the required information, let us not turn to the order parameter theory of the smectic dual shear superconductor [10]. Given the dislocation currents Eq. (3), the tangle of dislocation world lines can be described in terms of a single [9] Ginzburg-Landau-Wilson (GLW) order parameter field $\Psi$ as,

$$
\mathcal{L}_{\mathrm{GLW}}=\frac{1}{2}\left|\left(\partial_{\mu}-i b^{a} B_{\mu}^{a}\right) \Psi\right|^{2}+V(\Psi)+\mathcal{L}_{\text {gauge }}
$$

With the usual potential term $V(\Psi)=\frac{\lambda}{2}\left(|\Psi|^{2}-\Omega^{2} / \mu\right)^{2}$ and the Maxwell-like term $\mathcal{L}_{\text {gauge }}$ defined by Eq. (1). In the dislocation condensate amplitude has condensed, $\left|\Psi_{0}\right|=$ $\Omega / \sqrt{\mu}$, and by integrating out the dislocation phase field the remaining Higgs term can be written in gauge invariant form as [19],

$$
\mathcal{L}_{\text {Higgs,bare }}=\frac{1}{2} \frac{\Omega^{2}}{\mu} \frac{\left(b^{a} \sigma_{\mu}^{a}\right)\left(b^{b} \sigma_{\mu}^{b}\right)}{\partial_{\mu}^{2}},
$$

with $\partial_{\mu}^{2}=\frac{1}{c_{T}^{2}} \partial_{\tau}^{2}+\partial_{i}^{2}$, using the condensate velocity $c_{T}$. The glide constraint has still to be imposed and this is straightforwardly accomplished by adding a Lagrange multiplier to the action,

$$
\mathcal{L}_{\text {glide }}=i \lambda \varepsilon_{\tau \mu a} J_{\mu}^{a},
$$

which implies that the gauge fields in Eq. (4) should acquire an additional piece $B_{\mu}^{a} \rightarrow B_{\mu}^{a}+\lambda \varepsilon_{\tau \mu a}$, or, equivalently, that the dual stresses turn into $\sigma_{\mu}^{a} \rightarrow \sigma_{\mu}^{a}+$ $\delta_{\mu a} \partial_{\tau} \lambda-\delta_{\mu \tau} \partial_{a} \lambda$. Integrating out the multiplier field $\lambda$ 
we obtain a Higgs term respecting the glide constraint

$$
\mathcal{L}_{\text {Higgs }}=\frac{1}{2} \frac{\Omega^{2}}{\mu} \frac{\left(\sigma_{H}\right)^{2}}{\left(\partial_{\mu}^{(\eta)}\right)^{2}},
$$

with the dislocation second sound governed by

$$
\left(\partial_{\mu}^{(\eta)}\right)^{2}=\frac{1}{c_{T}^{2}} \partial_{\tau}^{2}+b^{a} \partial_{a}^{2} \sim \frac{1}{c_{T}^{2}} \omega_{n}^{2}+q^{2} \cos ^{2} \eta
$$

showing that the condensate only propagates along the direction of the Burgers vectors, introducing also the angle $\eta$ keeping track of the direction of the liquid direction relative to the wave vector. In addition, only the following stress component appears in the Higgs term,

$$
\sigma_{H}=\epsilon_{a c} b^{a} b^{b} \sigma_{c}^{b},
$$

which becomes in the quantum smectic $\sigma_{H}=\sigma_{y}^{x}$, taking the liquid direction along $\hat{x}$ such that $b^{b} b^{c}=\delta_{b, x} \delta_{c, x}$. The remainder is a lengthy but straightforward exercise: Eqs. (1) and (7), are expressed in the stress gauge fields via Eq. (2), followed by imposing gauge transversality (using a Lorentz gauge $\frac{1}{c_{T}^{2}} \partial_{\tau} B_{\tau}^{a}+\partial_{i} B_{i}^{a}=0$ ) and the Ehrenfest constraint. Finally, stress-strain relations are used to express the strain (phonon) propagators $\langle\langle\partial u \mid \partial u\rangle\rangle$ in terms of stress photon propagators $[9,14]$ and the spectral functions of the former are shown in Figs. 1 and 2.

We are now in the position to discuss some of the deeper issues related to the mode spectrum, Figs. 1 and 2. There are three propagating modes: the two (gauge-transversal) stress photons of the crystal plus the single "longitudinal" (or condensate) photon. For arbitrary $\eta$ 's the mode couplings conspire to produce the two massless "phonons" and the massive shear photon of Fig. 2. The longitudinal photons decouple completely from the condensate in the liquid and crystal directions of Fig. 1, but this is different in the transversal sectors: in the solid direction only the Higgsed transversal phonon shows up, while the "longitudinal photon" cannot be excited because of the onedimensional (glide) nature of the shear screening currents. In the liquid direction this is different: the massive mode is the massive second sound of the condensate (longitudinal photon), while the quadratic mode is like a Higgsed gauge particle where the "Higgs mechanism failed at the last moment".

If quantum smectics exist, are its modes observable? In Figs. 1 and 2 we show phonon propagators, but there is no obvious "atomistic" candidate quantum smectic. In electronic systems, the smectic modes can only be detected by their electromagnetic response, a story by itself which we will discuss elsewhere [20]. What about dc properties? The dual shear superconductor deals with dimensionality in a strange way: the dislocations form an ordered $(2+1) \mathrm{D}$ Higgs condensate, carrying only one-dimensional shear screening currents because of the glide principle. On "our side" of the duality this has the strange consequence that it would actually be a superfluid but only precisely along the liquid direction $[9,10]$. This is, however, a pathology of the (implicit) assumption governing the field theory that all length scales are large compared to the lattice constant. This is equivalent to the assumption that the single-particle (interstitial and vacancy) defects can be ignored, which is not the case for any finite lattice constant [9]. A real life Bose quantum smectic should therefore behave as an anisotropic two-dimensional superfluid.

We acknowledge helpful discussions with E. Demler, S. I. Mukhin, and Z. Nussinov. This work was supported by the Netherlands foundation for fundamental research of Matter (FOM).

*Electronic address: vladimir@lorentz.leidenuniv.nl

${ }^{\dagger}$ Electronic address: jan@lorentz.leidenuniv.nl

[1] P.G. de Gennes and J. Prost, The Physics of Liquid Crystals (Oxford University, New York, 1995), 2nd ed.

[2] P. M. Chaikin and T.C. Lubensky, Principles of Condensed Matter Physics (Cambridge University Press, Cambridge, England, 1995).

[3] S. A. Kivelson, E. Fradkin, and V. J. Emery, Nature (London) 393, 550 (1998).

[4] V. J. Emery, E. Fradkin, S. A. Kivelson, and T.C. Lubensky, Phys. Rev. Lett. 85, 2160 (2000).

[5] A. H. MacDonald and M. P. A. Fisher, Phys. Rev. B 61, 5724 (2000).

[6] R. Savit, Rev. Mod. Phys. 52, 453 (1980).

[7] H. Kleinert, Gauge Fields in Condensed Matter, Superflow and Vortex Lines, Disorder Fields, Phase Transitions (World Scientific, Singapore, 1989), Vol. I.

[8] B. I. Halperin and D. R. Nelson, Phys. Rev. Lett. 41, 121 (1978); D. R. Nelson and B. I. Halperin, Phys. Rev. B 19, 2457 (1979); A. P. Young, Phys. Rev. B 19, 1855 (1979).

[9] J. Zaanen, Z. Nussinov, and S.I. Mukhin, Ann. Phys. (N.Y.) 310, 181 (2004).

[10] V. Cvetkovic, Ph.D. thesis, Leiden University, 2006.

[11] P. C. Martin, O. Parodi, and P. S. Pershan, Phys. Rev. A 6, 2401 (1972).

[12] M.P. A. Fisher, P. B. Weichman, G. Grinstein, and D. S. Fisher, Phys. Rev. B 40, 546 (1989), and references therein.

[13] J. Hove and A. Sudbø, Phys. Rev. Lett. 84, 3426 (2000), and references therein.

[14] V. Cvetkovic and J. Zaanen, cond-mat/0511586.

[15] J. Friedel, Dislocations (Pergamon, New York, 1954); F. R. N. Nabarro, Theory of Dislocations (Clarendon, New York, 1967).

[16] F. A. Bais and C. J. M. Mathy, cond-mat/0602201; condmat/0602209; cond-mat/0602215.

[17] H. Kleinert, Gauge Fields in Condensed Matter, Stresses and Defects, Differential Geometry, Crystal Defects (World Scientific, Singapore, 1989), Vol. II.

[18] V. Cvetkovic, Z. Nussinov, and J. Zaanen, Philos. Mag. B 86, 2995 (2006).

[19] H. Kleinert and J. Zaanen, Phys. Lett. A 324, 361 (2004).

[20] V. Cvetkovic, S.I. Mukhin, and J. Zaanen (to be published). 